\title{
Evidence-Based Mental Health Programs in Schools: Barriers and Facilitators of Successful Implementation
}

\author{
Audra K. Langley $\cdot$ Erum Nadeem • \\ Sheryl H. Kataoka • Bradley D. Stein • \\ Lisa H. Jaycox
}

Published online: 11 May 2010

(C) The Author(s) 2010. This article is published with open access at Springerlink.com

\begin{abstract}
Although schools can improve children's access to mental health services, not all school-based providers are able to successfully deliver evidence-based practices. Indeed, even when school clinicians are trained in evidence-based practices (EBP), the training does not necessarily result in the implementation of those practices. This study explores factors that influence implementation of a particular EBP, Cognitive Behavioral Intervention for Trauma in Schools (CBITS). Semi-structured telephone interviews with 35 site administrators and clinicians from across the United States were conducted 6-18 months after receiving CBITS training to discuss implementation experiences. The implementation experiences of participants differed, but all reported similar barriers to implementation. Sites that successfully overcame such barriers differed from their unsuccessful counterparts by having greater organizational structure for delivering school services, a social network of other clinicians implementing CBITS, and administrative support for implementation.
\end{abstract}

A. K. Langley $(\bowtie) \cdot$ E. Nadeem · S. H. Kataoka

Division of Child and Adolescent Psychiatry, UCLA Semel Institute for Neuroscience and Human Behavior, 300 UCLA

Medical Plaza, Suite 1315, Los Angeles, CA 90095, USA

e-mail: alangley@mednet.ucla.edu

E. Nadeem

Division of Mental Health Services and Policy Research, New York State Psychiatric Institute, Columbia University, New York, NY, USA

B. D. Stein

University of Pittsburgh School of Medicine,

Pittsburgh, PA, USA

B. D. Stein · L. H. Jaycox

RAND Corporation, Santa Monica, CA, USA
This study suggests that EBP implementation can be facilitated by having the necessary support from school leadership and peers.

Keywords Child · Adolescent $\cdot$ School · Implementation · EBP

\section{Introduction}

Children with mental health needs do not commonly receive services for those needs (Flisher, 1997; Kataoka et al., 2003). Three-fourths of children who do receive services for mental health problems receive their care through the education sector, with schools being the most common point of entry for accessing mental health services (Farmer, Burns, Phillips, Angold, \& Costello, 2003). In light of this, the President's New Freedom Commission Report on Mental Health recommends "improving and expanding school mental health programs" as a means of improving access to mental health services (New Freedom Commission on Mental Health, 2003). Delivering mental health services through the school system, then, can address key financial and structural barriers that often prevent children from receiving needed services for mental health problems (Garrison, Roy, \& Azar, 1999).

Many schools already provide a variety of social-emotional support services, with an estimated majority of U.S. schools $(63 \%)$ providing prevention services, 59\% providing programs for behavioral problems, and approximately $75 \%$ of schools having school-wide programs supporting safe and drug-free schools (Foster, Rollefson, Doksum, Noonan, \& Robinson, 2005). However, schools vary in the organizational models that they use in delivering these services to students. Approximately one-half of 
U.S. schools use only school or district personnel to provide mental health services to students on campus, $23 \%$ combine school and district personnel with outside providers, and the remaining schools exclusively rely on outside community providers for student mental health services (Foster et al., 2005).

Despite existing infrastructure to deliver mental health services within schools, little is known about the quality of services being provided. National policies encourage the dissemination of evidence-based prevention and early intervention services in schools (Atkins et al., 1998; Trickett \& Birman, 1989; US Public Health Service, 2000), yet researchers have found that programs are frequently implemented unsuccessfully or with poor quality (Atkins, Frazier, Adil, \& Talbott, 2003). Further research is needed to improve implementation of school-based evidence-based programs, since successful implementation has been associated with improved outcomes for youth (Weist, 1997).

Applying Rogers' diffusion of innovations model (Rogers, 1995) to mental health services, Schoenwald and Hoagwood (2001) identify the following factors as some of the key dimensions in the implementation and dissemination of child mental health services: intervention characteristics, practitioner characteristics, client characteristics, service delivery characteristics, organizational characteristics, and service system characteristics. By addressing some of these factors, some intensive community-based programs (i.e., Multisystemic Therapy [MST] for delinquent youth [Borduin et al., 1995], Multidimensional Treatment Foster Care [MTFC] for children in out-of-home placement [Chamberlain, Leve, \& Degarmo, 2007]) have produced outcomes important to communities and have been successfully disseminated. Organizational culture and climate have also been shown to affect the success of mental health service programs in child service sectors (Esposito, 1999).

In contrast to organizational climate that focuses on aggregate perceptions of working in an organization, implementation climate emphasizes the perception of the importance of an intervention within the organization and the level of leadership and management support there is for implementing the intervention. In studying the implementation of specific mental health interventions in schools, it is the implementation climate that is likely more salient, although less well studied, in child mental health services, than the organizational climate of an entire school. Others have suggested that organizational factors, such as school structure, administrative leadership, school norms and policies, and the existing resources at the school are relevant to delivering mental health and educational interventions in schools (Atkins et al., 2003; Hoy, 1996; Trickett \& Birman, 1989). Thus, although the broad range of dimensions identified by Schoenwald and Hoagwood are relevant to the implementation of mental health services in schools, school organizational factors may be especially important to study in order to address barriers to implementing services in this setting.

To better explore and understand the potential barriers and facilitators to successful implementation of schoolbased interventions, this study reports on the findings from interviews conducted with providers with a range of implementation experiences of an established EBP, the Cognitive Behavioral Intervention for Trauma in Schools (CBITS; Jaycox, 2004; Kataoka et al., 2003; Stein et al., 2003). The goal of the interviews was to identify facilitators and barriers to successful implementation and to examine differences in implementation experiences between participants effectively implementing CBITS in schools and those who were not able to effectively implement the CBITS program. The current study seeks to augment this field of study by increasing the understanding of barriers specific to the school setting that may inhibit the uptake of evidence-based practice.

\section{Method}

\section{Participants}

Recruitment of interviewees began with brief telephone interviews with the directors of each of 8 sites that had previously received training in CBITS in the 6-24 months prior to data collection. All 8 directors agreed to participate in the initial interview, during which they were asked to nominate site clinicians who had implemented at least one CBITS group, defined in the current study as "successful implementers," and clinicians who had not completed a CBITS group, defined as "non-implementers", to be interviewed. For one site, which is part of our collaborative team, we e-mailed invitations to all clinicians who had been trained to participate $(N=17)$, and all those who responded were interviewed $(N=12)$. Due to differences in implementation success, some sites had all successful implementers (all trainees successfully implemented) and some had no successful implementers. Interviews occurred over the period between February and May 2007.

In all, 35 individuals were interviewed over the telephone (27 school mental health clinicians and 8 program directors; 9 men, 26 women) representing 8 sites involved in the implementation of CBITS. Of the 27 clinicians, 18 were "successful implementers" and 9 were "non-implementers." Participant clinicians were working primarily with middle school youth (grades 6-8) and included 10 (37\%) Licensed Clinical Social Workers (LCSWs), $4(15 \%)$ Marriage Family Therapists, 4 (15\%) Masters level School Psychologists, 2 (7\%) PhD Level School 
Psychologists, 2 (7\%) Clinical Psychologists, 2 (7\%) Master's Level Psychologists, 1 (4\%) Licensed Professional Counselor, 1 (4\%) School Nurse, and 1 (4\%) Bachelor's level counselor.

Including directors, 16 participants were drawn from 2 sites that utilized school district-employed clinicians, 5 participants were drawn from 3 sites that involved community mental health agencies co-locating in the schools, 8 participants were drawn from a site that had a collaborative partnership between community mental health agencies and school district-employed clinicians who co-lead CBITS, and 6 participants were drawn from 2 university-based sites, one of which involved groups being led by both school-employed clinicians and a university graduate student (3 participants), and the other that consulted with school-based systems of care clinicians (3 participants).

Cognitive Behavioral Intervention for Trauma in Schools (CBITS)

CBITS (Jaycox, 2004) is a school-based group intervention demonstrated to reduce symptoms of PTSD and depression among middle school children (ages 11-15) exposed to traumatic events (Kataoka et al., 2003; Stein et al., 2003). It incorporates core skill-based components of cognitive behavioral therapy (CBT) including psychoeducation, relaxation, cognitive coping, trauma narrative and processing of traumatic memories and grief, gradual in vivo mastery of trauma reminders and generalized anxiety, and social problem-solving. These skills are learned, processed, and practiced in 10 group sessions and 1-3 individual meetings. Between sessions, children participate in activities and practice assignments that reinforce the skills they learn in session. CBITS also includes parent and teacher education sessions. Typically, children attend CBITS sessions weekly during the school day for approximately 10 weeks.

Resulting from a community-research partnership, the CBITS intervention was designed in collaboration with school stakeholders specifically for delivery in schools with several key adoption characteristics in mind: relative advantage over usual care practice, compatibility with other behaviorally oriented practices in schools, and lessons that were developed in collaboration with schoolbased clinicians to minimize complexity.

The process of identifying students for the CBITS program typically involves obtaining parent permission for screening in a general school population (students in regular classrooms), administering the screener to the group of students with parent permission, identifying students with elevated symptoms and forming groups for the intervention, and obtaining permission for the groups. Thus, there are several logistical steps involved in getting the groups up and running, in addition to running the groups themselves.

All study participants participated in the standard 2-day CBITS training provided by CBITS training faculty, which includes: (1) An overview of child trauma and PTSD and the mental health and academic consequences, (2) A review of the history and evidence base of CBITS, (3) Thorough session by session demonstrations and supervised dyadic and small group practice of each core concept for child group and individual sessions, including how to make the material culturally and contextually relevant to the audience, (4) Review of parent and teacher sessions, and (5) Engagement activities around implementation issues and site planning.

Procedure

Director interviews consisted of 19 questions, "nonimplementer" interviews included 17 probes, and "successful implementer" interviews consisted of 49 items that included the same items as the non-implementer interview as well as questions about running the groups. Both clinician semi-structured interviews asked participants about their training and professional background as well as (1) familiarity with other clinicians implementing CBITS, (2) planned frequency of CBITS delivery in the future on a 10-point scale ranging from "never" to "in most cases," and (3) barriers encountered in attempting to deliver CBITS. In addition, clinicians were also asked to rate the actual or perceived level of difficulty of the CBITS intervention on a scale of 1-10, 1 being "not easy at all" and 10 being "very easy". Those who had successfully implemented at least one CBITS group were also asked about (1) implementation support, (2) clinical support or consultation they would find most useful during CBITS implementation, and (3) funding for CBITS implementation.

After obtaining verbal consent, telephone interviews lasted an average of $30 \mathrm{~min}$, ranging from approximately 20-45 min, and participants received a \$40 gift card for participating in the study. The RAND and UCLA Institutional Review Boards approved all study procedures.

\section{Analysis}

Extensive field notes and selective quotations for all interviews were recorded on the semi-structured interview form. Quantitative and qualitative data from the interviews were then reviewed to explore general topics that arose in the interviews. Major domains of inquiry based on common themes in the implementation literature were preliminarily coded using qualitative data analysis software (ATLAS.ti). Subsequently, the research team discussed the 
content of each domain and refined the coding scheme by expanding, collapsing, or eliminating codes until there was a refined list of mutually agreed upon codes that tapped into implementation barriers, implementation supports, perceptions about the intervention, models of service delivery, and clinical supports. Additional coding was done based on the range of responses within each domain. Using the software's filtering and grouping tools, clinicians in the "successful implementer" category $(n=18)$ were compared to clinicians in the "non-implementer" category $(n=9)$ with regard to specific implementation barriers, perceptions about the intervention (e.g., ease of use), mental health services model employed (e.g., partnered implementation, school-employed clinicians only), implementation support (e.g., funding, knowing others who were implementing the same intervention), and specific clinical supports.

\section{Results}

Table 1 provides information about the implementer and non-implementer clinicians participating in the interviews. Clinicians from 4 of the 8 training sites participating had school-community mental health agency partnerships and all reported successful implementation of at least one CBITS group. The two school sites that employed their own school-based mental health clinicians had a lower percentage of trained clinicians successfully implement CBITS. Among the two university programs that partnered with schools, one had all their clinicians successfully implement CBITS, while the other had no implementers.

Three broad themes consistently emerged across interviews: (1) Implementation Barriers, (2) Availability of Implementation Support, and (3) Perceptions of the Program. In some cases, these themes differed across the implementer and non-implementer groups, and in other cases, there were no differences. The frequency of each implementation barrier reported was totaled and rank ordered separately for successful implementers and nonimplementers. Table 2 provides a rank-ordered list of the top 4 implementation barriers for successful implementers versus non-implementers.

\section{Implementation Barriers}

The four main barriers to the implementation of CBITS included competing responsibilities, parent engagement,

Table 1 Number of implementing and non-implementing clinicians represented in the sample and region served, sorted by site and type of service delivery model

\begin{tabular}{|c|c|c|c|}
\hline \# of Clinicians interviewed by site & $\begin{array}{l}\text { \# }(\%) \text { of Successful } \\
\text { implementers }\end{array}$ & \# of Non-implementers & $\begin{array}{l}\text { Urban/Suburban/Rural } \\
\text { (Region) }\end{array}$ \\
\hline \multicolumn{4}{|l|}{ School-employed } \\
\hline 12 & $6(50 \%)$ & 6 & Urban (West) \\
\hline 1 & $0(0 \%)$ & 1 & Rural (West) \\
\hline \multicolumn{4}{|c|}{ Community or mental health agency partnering with schools } \\
\hline 1 & $1(100 \%)$ & 0 & Urban (Midwest) \\
\hline 1 & $1(100 \%)$ & 0 & Urban (East) \\
\hline 7 & $7(100 \%)$ & 0 & Suburban (Midwest) \\
\hline 1 & $1(100 \%)$ & 0 & Urban (West) \\
\hline \multicolumn{4}{|l|}{ University partnering with schools } \\
\hline 2 & $2(100 \%)$ & 0 & Rural (Mountain) \\
\hline \multicolumn{4}{|c|}{ University consulting with systems of care } \\
\hline 2 & $0(0 \%)$ & 2 & Urban (Midwest) \\
\hline
\end{tabular}

Table 2 Rank order of top 4 implementation barriers for successful implementers versus non-implementers

\begin{tabular}{ll}
\hline Successful implementers & Non-implementers \\
\hline 1. Lack of parent engagement & 1. Competing responsibilities \\
2. Competing responsibilities & $2-3$. Lack of parent engagement \\
3. Logistical barriers & $2-3$. Logistical barriers \\
4. Lack of support from school administrators and teachers & 4. Lack of support from school administrators and teachers \\
\hline
\end{tabular}


logistics, and support from administrators and teachers (see Table 2). These themes were discussed by all participants, and the issues raised did not vary between clinicians who were successful CBITS implementers and those who were non-implementers.

\section{Competing Responsibilities (Ranked \#2 \\ by Implementers and \#1 by Non-Implementers)}

Clinicians described having multiple duties on campus other than implementing CBITS. For example, one clinician described having "no time to run the groups." Others described having limitations in scheduling and coordinating with school staff. One clinician indicated that she had a plan to implement CBITS, but cited "never getting around to scheduling, getting space, having the same schedule as the school clinician partner." One of the few successful implementers at a school-employed site stated that "It was tough, but despite all the barriers, I just made it work. I foresee numerous problems for those who do not have my scheduling flexibility or principal support. They won't have time." Another successful implementer described that she got around the barrier of having time to screen students for inclusion in CBITS groups "by having 5 people, including agency and school support staff, assist." Successful implementers from mental health agencies, who were co-facilitating groups with a school-employed clinician, reported fewer competing responsibilities than school-employed clinicians overall. Similarly, the agency clinician was able to manage some of the duties that the school-employed clinician may not have had time for, “...preparing copies of handouts, bringing little prizes, and continuing with the group if I [the school-employed clinician] was pulled away for something 'urgent'."

\section{Lack of Parent Engagement (Ranked \#1 \\ by Implementers and \#2-3 by Non-Implementers)}

Many clinicians described difficulties in contacting parents. One clinician described clear difficulty in "reaching parents" and working with parents of impoverished students such as"migrant agricultural workers who work $12 \mathrm{~h}$ days. [They] weren't necessarily resistant but couldn't get in touch." Clinicians who implemented CBITS also described challenges in engaging parents in treatment. For example, one clinician reported that "It [the main barrier] was parent participation. We had only one parent session and parents did not help kids with getting their practice and homework done". In response, this clinician modified homework with parents by "doing practice at the beginning of each session whenever possible" with participating students.
Logistical Barriers (Ranked \#3 by Implementers and \#2-3 by Non-Implementers)

Many participants discussed the challenge that the "school environment can be hectic and crisis driven." For many nonimplementers, the difficulties of acquiring space and finding time in the school environment where groups could be conducted appeared insurmountable and dissuaded many from trying to move forward. Successful implementers reported having comparable challenges with the hectic school environment, citing difficulties " $\mathrm{getting}$ kids to group on time. Because they were late it was hard [for students] to grasp all session material in a shorter period of the time." One mental health agency clinician who was co-facilitating a CBITS group with a school-employed clinician found that, "school[-employed] partners are good at organizing space, working with student schedules, pulling students, and knowing who to talk to to get a question answered."

Another them, was teachers not allowing children to leave class or other school activities. For example, one clinician described, "Teachers don't want to let kids out of class. Assemblies, special schedules, consolidated days make scheduling difficult. [A] fire drill in the middle of the relaxation exercise group (was also disruptive)".

\section{Lack of Support from School Administrators} and Teachers (Ranked \#4 by Implementers and \# 4 by Non-Implementers)

Several successfully implementing clinicians stated that implementation would not have been possible without support from the school principal. "We have great buy in and support from principal and support staff, even teachers too." Another discussed that the "best advice I got was to find a good principal. It all depends on if the school is ready and open to the program." Several successful implementers commented on the relevance of this type of higher level support to their success in overcoming other potential barriers. Successful implementers also observed that "if a school facilitator or partner was excited", they would be more likely to "do the groundwork with teachers, staff, students and parents; then group went much easier" and that getting buy-in from teachers is key to overcome the logistical barrier of pulling them from class. For nonimplementers, the perceptions that school administration and/or teachers were not invested in the program were barriers to moving forward with implementation.

Availability of Implementation Support

In contrast to the implementation barriers, which were very similar across successful implementers and non-implementers, there were differences observed between the two 
groups in terms of the availability of implementation support.

\section{Professional Network}

Among the successful implementers of CBITS, all reported knowing someone else who was conducting CBITS groups, either within their own school, or within their organization or region. Conversely, none of the non-implementers knew other people who were implementing CBITS.

\section{Funding}

All successful implementers were from sites that had grant funding or some funds set aside by the management or administration for CBITS implementation. However, there was one site with clinicians who did not implement CBITS despite having specific grant funding to do so.

Perceptions of the Program

\section{Level of Difficulty of the CBITS Intervention}

When asked to rate how easy it was/would be to conduct a CBITS group on a scale from 1 to 10 with 10 being "very easy", $78 \%$ of the respondents from the successful implementer group responded in the 7-9 range (Mean $=7.8$ ). Similarly, non-implementers perceived that running the groups would be easy, with $85 \%$ reporting ratings $7-9$ (Mean = 7.0). Clinicians described the CBITS manual as "Laid out well for you and planning time was minimal, with quick and easy modifications." The manual "makes it easy" with "user-friendly material." One implementer noted that the "curriculum is so well-defined and easy to plan... that there is] very little prep needed."

\section{Future Use of Program Elements}

A majority of the CBITS successful implementers (90\%) planned to use CBITS techniques in the future in "most cases" for students who experienced trauma, while the plans of non-implementers to use CBITS were more mixed, ranging from "never" to "in most cases." It is important to note that the successful implementers answered this question after having had prior implementation experience with CBITS, while the non-implementers did not.

Model of Clinical Consultation among Successful Implementers

Implementers were asked about what type of clinical support was available to them and what types of clinical support would be preferred by them during implementation. All successful implementers had consultation regularly available, even if it was just having someone available to consult with on an as-needed basis. A majority of implementers rated consultation as very useful (61\%). Most implementers described a preference for monthly-in- person consultation meetings and some endorsed monthly group phone consultation, many had other specific ideas in mind. Some had or would have liked to have had "weekly on-site individual supervision/consultation or weekly phone consultation" and "specific support about implementation issues (i.e., help with screening students for the program)." Many felt that it benefitted them to hear from others who were implementing and "liked how there were others at differing stages on the [consultation] call”. However, some implementers reported that "1:1 consultation or smaller groups are better than larger groups, especially if on a conference call." Implementers from one site (where all trainees successfully implemented) appreciated "accessibility [of the consultant] on an as needed basis-this was always available and she was very responsive." Implementers also reported that having a "mid-way in-person check-in with other implementing clinicians and a supervisor was helpful." Finally, implementers commented that specific help focused on the more difficult sessions would be beneficial.

\section{Discussion}

Given the importance of schools in improving access to evidence-based practices (EBPs) for children, this study sheds new light on important variables that may facilitate or hinder implementation of EBPs within the school setting. We found a number of important implementation barriers (i.e., competing responsibilities, logistics, parental consent, and administrator/teacher support) as well as facilitating factors (i.e., professional networks, financial resources). These insights from the direct experiences of providers echo themes that have been highlighted in systematic reviews and conceptual models of implementation in a variety of service settings such as support from administrators and other staff, implementation support and consultation, availability of resources (e.g., time, money, tangible supports), and perceptions about the intervention itself (e.g., ease of use, relevance, compatibility with the setting) (e.g., Fixsen, Naoom, Blasé, Friedman, \& Wallace, 2005; Greenhalgh, Robert, McFarlane, Bate, \& Kyriakidou, 2004; Klein and Sora, 1996; Schoenwald \& Hoagwood, 2001). Similar barriers and facilitators were found in a study of school-based treatment developers' perceptions of implementation barriers and facilitators (Forman, Olin, Hoagwood, Crowe, \& Saka, 2009). The current study also 
provides important information about the types of support and consultation models desired by school-based providers that have experience using an EBP.

Given that both implementers and non-implementers perceived that implementing the CBITS group protocol would be relatively easy, it does not seem like perceptions of the intervention was an implementation barrier in the case of CBITS. In addition, it makes sense that clinicians who have implemented an intervention and have more practice with the components of an intervention would report being more likely to use such components in the future, versus those who have not yet been able to implement, since they have established experience and comfort level with the materials. Rather, the major barriers to successful implementation appeared to at the systems and organizational levels.

Competing responsibilities emerged as the strongest barrier to program implementation, with all non-implementers endorsing it. It was endorsed as the second most frequent implementation barrier by successful implementers, and is consistent with prior research (Forman et al., 2009). Its prominence in this study highlights the importance of this barrier to the experience of frontline clinicians in school settings. For non-implementing clinicians, competing responsibilities appear to ultimately be the reason that they were unable to successfully implement. Subsequently, they may not have identified some of the other barriers (i.e., parent engagement, logistics, administrator, and teacher buy-in) as frequently because they had not gotten far enough along in the implementation process to experience them.

Interestingly, community clinicians who co-located onto a school campus (a partnership between mental health/community agency and schools) reported fewer competing responsibilities while on the school grounds than their school-employed counterparts. This may reflect the fact that they could focus on implementation with less likelihood of being pulled away for another school responsibility or crisis. These successful implementers from a mental health agency who co-located onto campus to run groups with a school-employed clinician reported being able to support implementation by bringing the snacks or materials for the groups and providing copies of worksheets, handouts, and screeners to decrease time and financial burden to school-employed clinicians and support staff. On the other hand, the school-employed clinician appeared to be more able to successfully support the logistical aspects of working and scheduling within the school building and calendar. In addition to capitalizing on opportunities for partnership to aid implementation success, the importance of competing responsibilities also highlights a need for school-based clinicians' roles to be redefined in such a way to allow time for the CBITS groups and their formation. This was a critical step for those who were able to successfully implement, and caused difficulties in the cases where it did not happen. This type of realignment of staff has been identified by Fixsen et al. (2005) as one of the elements of program installation as an organization seeks to implement a new practice.

Insufficient teacher buy-in was ranked as the fourth highest barrier among successful implementers of CBITS. Clinician's perceived teacher buy-in as affecting implementation of CBITS groups since teachers who were not supportive of CBITS or who did not perceive benefits of the program were more reluctant to let their students leave class for the group. This perception is in line with research and models of school-based implementation, which highlight the importance of the support of teachers and administrators to successful implementation (Domitrovich et al., 2008; Forman et al., 2009). Pre-implementation activities designed to increase buy-in among administrators, school staff, and parents are an important part of a program's implementation. To this end, a multimodal strategy to increase the awareness of the problem being targeted and its impact on students may be called for, and can include teacher in-services, parent meetings, and community and stakeholder focus groups. As part of this process, there may be additional value in using community partnerships between clinicians, school staff, community organizations, and parents to engage communities and address ongoing implementation issues (Ngo et al., 2008). It may be that within the process of establishing a school mental health partner relationship, some of this groundwork is taking place in a way that it may not be in a system using internal school-employed clinicians where relationships are already established.

Teacher concerns about issues like pulling students out of class also contributed to the logistical concerns barrier, endorsed by a majority of both implementers and nonimplementers with regard to difficulty with some teachers letting kids leave class, while it also speaks to difficulty finding space, making copies, and other pragmatic issues. Logistical concerns are important to implementation across a variety of settings (Fixen et al., 2005; Greenhalgh et al., 2004). Their presence in the current study as one of the four major barriers highlights the unique and complex challenges inherent to implementing in a non-specialty mental health setting such as schools.

The final major barrier to implementation was obtaining parent permission. In the sites that successfully implemented CBITS, groups were run with the subset of students whose parents had provided permission, despite clinicians' sense that they were missing a number of other students that could benefit. They reported, however, that they may have identified and included more youth if they had gotten 
better consent rates and had an easier time getting in contact with parents during groups. Parent engagement in school-based services has been a consistent challenge in the implementation of school mental health programs more broadly (Weist, Evans, \& Lieber, 2003), and it is not surprising that parental involvement was a challenge here. The development of strategies for engaging parents in schoolbased mental health services like CBITS may be a key element in increasing access to quality mental health services for youth in schools.

Among the sites interviewed in this study, schools partnering with mental health agencies appeared to be more likely to experience successful implementation of an EBP. All four of the sites who had this arrangement were able to successfully implement CBITS. Establishing partnerships between schools and mental health agencies appear to lead to a greater ability to surmount the inherent challenges in school-based mental health provision, thereby distinguishing sites that were able to implement the program from those that were not. Joint resources and expertise may enhance the capacity of providers across both settings to manage the competing demands and logistic challenges.

Successful implementers were all from sites with some degree of grant funding that enabled them to have devoted time to implement CBITS. Although the frontline clinicians interviewed in this study did not explicitly mention funding as a barrier, this pattern is a reflection of Forman et al.'s (Forman et al. 2009) findings in which treatment developers cited funding as the most frequently cited obstacle to successful implementation. Likewise, Aarons, Wells, Zagursky, Fettes, \& Palinkas (2009) found among multiple stakeholders implementing EBPs in community mental health agencies that funding was endorsed as both the most important and least changeable factor in implementation success. With grant funding, often comes dedicated time and resources that would reduce the barrier of competing responsibilities described above. It may be that such funding is necessary, yet not sufficient factor, to successful implementation.

Beyond the surmounting of barriers, familiarity with others implementing the same program clearly distinguished implementers from non-implementers. This may be a facilitator in terms of perceived buy-in from peers and the organization. Moreover, providers may experience a greater sense of support if they know that they can confer with another implementer if needed. Finally, although the model for clinical support or consultation varied from site to site, all successful implementers had some form of clinical support or consultation in place that a majority found to be quite helpful. The forethought of understanding the importance of and creating a plan for clinical support and consultation may reflect an organizational characteristic that facilitates implementation success. The fact that most successful implementers preferred group consultation meetings highlights the importance of both the availability of consultation support itself as well as the aforementioned ability to connect, learn from, and share ideas with others who are also engaged in the implementation process. Interestingly, some noted this venue to be best in-person, especially with larger groups, so group size is an important factor to consider when planning for consultation. Likewise, the notions of having consultation available on an as-needed basis and session-specific support for program content that clinicians may find challenging were notable ingredients that could be integrated into an informed plan for clinical support. Each of these findings makes the case for setting up consultation and supervision plans before and during training in EBP.

There are some limitations to the study that are important to consider. The clinicians interviewed for this study are not representative of all providers who have implemented an EBP in schools, and there may be some bias introduced by selecting them via nominations from their director. There are certainly school-community mental health agency partnerships and/or grant-funded programs that do not successfully implement EBPs in schools. Similarly, there may be additional cases of school-only and clinic-only programs that have been able to implement EBPs successfully. More focused study of these types of sites might illuminate additional strategies for allocating resources and addressing other challenges specific to these organizational structures. However, our findings provide useful insight into models of service delivery and conditions that may increase the likelihood of surmounting common implementation challenges. Finally, it is important to note that the current study examines provider experiences of a single EBP. There may be some results that are unique to CBITS. However, because the themes in our study are similar to those found in the broader implementation literature, the insights gleaned from this study may generalize to the implementation of other school EBPs.

Although common challenges exist among all sites attempting to adopt and implement an evidence program in the school setting, there are a few things that tend to distinguish those who are able to overcome barriers to implementation from those who are not. The service model for school-based implementation seems to be related to implementation, with more success when a school partners with a community or mental health agency. Instead of focusing on what specific barriers are, it may be relevant to shift the focus onto what sets of conditions make it more or less likely that barriers can be surmounted. 
Open Access This article is distributed under the terms of the Creative Commons Attribution Noncommercial License which permits any noncommercial use, distribution, and reproduction in any medium, provided the original author(s) and source are credited.

\section{References}

Aarons, G. A., Wells, R. S., Zagursky, K., Fettes, D. L., \& Palinkas, L. A. (2009). Implementing evidence-based practice in community mental health agencies: A multiple stakeholder analysis. American Journal of Publilc Health, 99(11), 2087-2095.

Atkins, M. S., Frazier, S. L., Adil, J. A., \& Talbott, E. (2003). Schoolbased mental health services in urban communities. In M. D. Weist (Ed.), Handbook of school mental health: Advancing practice and research. Issues in clinical child psychology. New York, NY: Kluwer Academic/Plenum Publishers.

Atkins, M. S., McKay, M. M., Arvanitis, P., London, L., Madison, S., Costigan, C., et al. (1998). An ecological model for school-based mental health services for urban low-income aggressive children. Journal of Behavioral Health Services and Research, 25(1), 64-75.

Borduin, C. M., Mann, B. J., Henggeler, S. W., Fucci, B. R., Blaske, D. M., \& Williams, R. A. (1995). Multisystemic treatment of serious juvenile offenders: Long-term prevention of criminality and violence. Journal of Consulting and Clinical Psychology, 63(4), 569-578.

Chamberlain, P., Leve, L. D., \& Degarmo, D. S. (2007). Multidimensional treatment foster care for girls in the juvenile justice system: 2-year follow-up of a randomized clinical trial. Journal of Consulting and Clinical Psychology, 75(1), 187-193.

Domitrovich, C. E., Bradshaw, C. P., Poduska, J. M., Hoagwood, K., Buckley, J. A., Olin, S., et al. (2008). Maximizing the implementation quality of evidence-based preventive interventions in schools: A conceptual framework. Advances in School Mental Health Promotion, 1, 6-28.

Esposito, C. (1999). Learning in urban blight: School climate and its effect on the school performance of urban, minority, low-income children. School Psychology Review, 28(3), 365-377.

Farmer, E. M., Burns, B. J., Phillips, S. D., Angold, A., \& Costello, E. J. (2003). Pathways into and through mental health services for children and adolescents. Psychiatric Services, 54(1), 60-66.

Fixsen, D. L., Naoom, S. F., Blasé, K. A., Friedman, R. M., \& Wallace, F. (2005). Implementation research: A synthesis of the literature. Tampa, Fl: University of South Florida, Louis de la Parte Florida Mental Health Institute, The National Implementation Research Network.

Forman, S. G., Olin, S. S., Hoagwood, K. E., Crowe, M., \& Saka, N. (2009). Evidence-based interventions in schools: Developers' views on implementation barriers and facilitators. School Mental Health, 1, 26-36.

Foster, S., Rollefson, M., Doksum, T., Noonan, D., \& Robinson, G. (2005). School mental health services in the United States,
2002-2003. DHHS Pub. No. (SMA) 05-4068. Rockville, MD: Center for Mental Health Services, Substance Abuse and Mental Health Services Administration.

Garrison, E. G., Roy, I. S., \& Azar, V. (1999). Responding to the mental health needs of Latino children and families through school-based services. Clinical Psychology Review, 19(2), 199-219.

Greenhalgh, T., Robert, G., McFarlane, F., Bate, P., \& Kyriakidou, O. (2004). Diffusion of innovations in service organizations: Systematic review and recommendations. The Milbank Quarterly, 82(4), 581-629.

Hoy, W. K. (1996). The organizational climate of middle schools. Journal of Education Administration, 34(1), 41-59.

Jaycox, L. H. (2004). Cognitive behavioral intervention for trauma in schools. Longmont, CO: Sopris West Educational Services.

Kataoka, S. H., Stein, B. D., Jaycox, L. H., Wong, M., Tu, W., Escudero, P., et al. (2003). A school-based mental health program for traumatized Latino immigrant children. Journal of the American Academy of Child and Adolescent Psychiatry, 42(3), 311-318.

Klein, K. J., \& Sora, J. S. (1996). The challenge of implementaion innovation. The Academy of Management Review, 21(4), $1055-1080$.

New Freedom Commission on Mental Health, Achieving the Promise: Transforming Mental Health Care in America. (2003). Final Report. DHHS Pub. No. SMA-03-3832. Rockville, MD.

Rogers, E. M. (1995). Diffussion of innovation. New York, NY: Free Press.

Shoenwald, S. K., \& Hoagwood, K. (2001). Effectiveness, transportability, and dissemination of interventions: What matters when? Psychiatric Services, 52(9), 1190-1197.

Stein, B. D., Jaycox, L. H., Kataoka, S. H., Wong, M., Tu, W., Elliott, M. N., et al. (2003). A mental health intervention for schoolchildren exposed to violence: A randomized controlled trial. Journal of the American Medical Association, 290(5), 603-611.

Trickett, E. J., \& Birman, D. (1989). Taking ecology seriously: A community development approach to individually based preventive interventions in schools. Thousand Oaks, CA: Primary Prevention and Promotion in the Schools. Sage Publications.

U.S. Public Health Service. (2000). Report of the surgeon general's conference on children's mental health: A national action Agenda. Washington, DC: Department of Health and Human Services.

Weist, M. D. (1997). Expanded school mental health services: A national movement in progress. Advances in Clinical Child Psychology, 19, 319-352.

Weist, M. D., Evans, S. W., \& Lever, N. A. (2003). Handbook of school mental health: Advancing practice and research. Issues in clinical child psychology. New York, NY: Kluwer Academic/ Plenum Publishers. 